Patricia Whitelock and Russell Cannon, eds.

\title{
Carbon Stars in the Sagittarius Dwarf Galaxy
}

\author{
Patricia Whitelock and John Menzies \\ SAAO, P O Box 9, Observatory, 7935, South Africa
}

Mike Irwin

RGO, Madingley Road, Cambridge CB3 OEZ, UK

Michael Feast

UCT, Rondebosch, 7700, South Africa

\begin{abstract}
Coordinates are listed for 26 carbon stars which are radial velocity members of the Sagittarius Dwarf Galaxy (SDG). Their nearinfrared properties indicate that most are on an extended Asymptotic Giant Branch (AGB), and the total number of such stars in the SDG is estimated at about 100. At least one-third of the $\mathrm{C}$ stars studied are large amplitude, i.e. Mira, variables; these are the first Miras to be found in a dSph galaxy. Surprisingly, the most luminous $\mathrm{C}$ star is not a Mira, but a low amplitude variable.

A comparison of the properties of $\mathrm{C}$ stars in the Bulge and in the SDG indicates that the two groups cannot be drawn from the same population. It is therefore highly unlikely that the Bulge $\mathrm{C}$ stars are, contrary to previous suggestions, related in any way to the SDG.
\end{abstract}

\section{Introduction}

By comparing the Sagittarius Dwarf Galaxy (SDG) giant branch with that of the globular cluster 47 Tuc, Whitelock et al. (1996, hereafter Paper I) showed that the SDG contained a significant population with a metallicity of around $[\mathrm{Fe} / \mathrm{H}]=-0.85$. They also examined the infrared colours of $26 \mathrm{C}$ stars or possible $\mathrm{C}$ stars, only 7 of which were then known to be radial velocity members of the dwarf galaxy ${ }^{1}$. They noted that the reddest and brightest of these seemed similar to the $\mathrm{C}$ stars in Fornax, while the less luminous examples were more like those from the more metal deficient systems of Sculptor, Draco and Ursa Minor.

This paper discusses the infrared colours of 26 stars which are spectroscopically confirmed carbon stars and members of the SDG. Eleven of these were in the sample of Paper I and the rest are new.

\footnotetext{
${ }^{1}$ Note that 4 of the 30 stars listed in Table 1 of Paper I were shown to be M stars in the Bulge in that paper.
} 


\section{Source Selection and Observations}

C star candidates were selected from matched pairs of UKST survey plates in the $O R / R$ and $B_{J}$ passbands. The plates were measured on the APM facility, and processed in the usual manner (e.g. Totten \& Irwin, 1998; Kunkel, Demers \& Irwin, 1997) to produce colour-magnitude diagrams for several selected regions in the main body of the SDG. Target areas included: $3^{\circ} .4 \times 3^{\circ} .4$ regions centred on the globular cluster M54 at the heart of the SDG and a comparable region $4^{\circ}$ east of this position, close to the location of the secondary "peak" visible in the isopleth map shown in Ibata et al. (1994); a $3^{\circ} .4 \times 3^{\circ} .4$ region around the globular cluster Arp2; and $2^{\circ} .5 \times 2^{\circ} .5$ regions around the globular clusters Ter 7 and Ter 8.

A well defined AGB is visible for the central regions of the SDG and this was used to define the selection boundaries, $B_{J}-R>2.5$ and $13.0 \lesssim R \lesssim 15.0$, within which cool N-type $\mathrm{C}$ stars at the distance of the SDG are expected to be located.

Follow-up spectroscopy was conducted at Las Campanas on the du Pont $2.5 \mathrm{~m}$ telescope using the MODular spectrograph (MODspec) and at SAAO on the $1.9 \mathrm{~m}$ telescope using the CCD spectrograph. C stars were distinguished from $\mathrm{K}$ and $\mathrm{M}$ stars using the strong $\mathrm{CN}$ bands around $8000 \AA$ and radial velocities were obtained for confirmed carbon stars by cross-correlating with standard star templates (see Totten \& Irwin 1998 for more details).

From the 73 stars examined, 26 were confirmed to be $\mathrm{C}$ stars with radial velocities matching the systemic velocity of the SDG, one was a $C$ star with Bulge kinematics and the others had spectra of $\mathrm{M}, \mathrm{K}$ or indeterminate type. The equatorial coordinates for the SDG C stars are given in Table 1.

Near-infrared, $J H K L$, photometry was obtained for all of these carbon stars at various epochs (see Paper I). The intention was to characterise the variability and determine pulsation periods where possible and therefore more observations have been obtained of the brighter and redder stars, which were thought to be better candidates for large amplitude variables.

\section{Total Number of $\mathbf{C}$ stars in Sagittarius}

We can make a provisional estimate of the total $\mathrm{N}$-type (i.e. intermediate age) carbon star population in the main body of the SDG by noting that the major axis profile of the SDG is relatively flat due to the ongoing effects of tidal disruption and extends over approximately $22^{\circ}$; the minor axis profile covers an effective scale size of about $7^{\circ}$. Our C star survey fields sample both the central and outer parts of the dwarf and cover $\sim 30$ per cent of the scale area of the main body of the SDG. From similar surveys in and around the Magellanic Clouds we expect a completeness of around 80 per cent for these fields (Kunkel et al. 1997). Hence we have sampled $\sim 25$ per cent of the $\mathrm{C}$ stars in the main body of the SDG. This implies that the total intermediate age SDG C star population in the main body of the dwarf, $\sim 100$ objects, is similar in number to that in the Fornax dwarf. 
Table 1. Carbon Stars in the SDG.

\begin{tabular}{|c|c|c|c|c|c|c|c|c|c|}
\hline \multirow{2}{*}{ No. } & \multicolumn{2}{|c|}{ RA } & \multicolumn{2}{|c|}{ (1950) } & \multicolumn{2}{|c|}{ Dec } & \multirow{3}{*}{$\begin{array}{c}\text { Paper I } \\
\text { name } \\
\text { C-1 }\end{array}$} & \multirow{2}{*}{$\begin{array}{l}\text { Var } \\
\text { type }\end{array}$} & \multirow{2}{*}{$\begin{array}{c}\mathrm{P} \\
\text { (day) }\end{array}$} \\
\hline & h & $\mathrm{m}$ & s & o & & $\prime \prime$ & & & \\
\hline 1 & 18 & 43 & 11.9 & -30 & 18 & 24 & & & \\
\hline 2 & 18 & 43 & 26.3 & -30 & 49 & 8 & UKST-3 & M & 300 \\
\hline 3 & 18 & 49 & 36.0 & -31 & 16 & 15 & UKST-9 & SR & \\
\hline 4 & 18 & 49 & 39.0 & -30 & 0 & 13 & C-3 & SR & \\
\hline 5 & 18 & 50 & 18.6 & -29 & 42 & 8 & C-2 & M & 228 \\
\hline 6 & 18 & 51 & 12.3 & -30 & 29 & 0 & UKST-12 & SR & 280 \\
\hline 7 & 18 & 51 & 28.0 & -29 & 58 & 8 & UKST-13 & & \\
\hline 8 & 18 & 53 & 42.4 & -31 & 28 & 40 & UKST-15 & SR & \\
\hline 9 & 18 & 54 & 9.3 & -30 & 52 & 0 & & & \\
\hline 10 & 18 & 54 & 11.6 & -29 & 11 & 56 & UKST-16 & & \\
\hline 11 & 18 & 55 & 1.9 & -30 & 59 & 16 & UKST-18 & SR & \\
\hline 12 & 18 & 55 & 19.5 & -30 & 51 & 45 & & & \\
\hline 13 & 18 & 58 & 2.5 & -31 & 30 & 25 & & & \\
\hline 14 & 18 & 58 & 17.0 & -30 & 16 & 30 & & & \\
\hline 15 & 18 & 58 & 41.0 & -30 & 37 & 0 & & M & 360: \\
\hline 16 & 18 & 59 & 58.7 & -30 & 7 & 2 & & & \\
\hline 17 & 19 & 1 & 36.2 & -31 & 15 & 27 & & M & 317: \\
\hline 18 & 19 & 6 & 28.4 & -30 & 1 & 50 & & SR & \\
\hline 19 & 19 & 8 & 1.4 & -31 & 58 & 10 & & SR & \\
\hline 20 & 19 & 8 & 54.0 & -31 & 43 & 35 & & & \\
\hline 21 & 19 & 12 & 38.2 & -32 & 56 & 34 & C-4 & & \\
\hline 22 & 19 & 20 & 8.5 & -34 & 6 & 6 & & M & \\
\hline 23 & 19 & 20 & 28.6 & -34 & 37 & 7 & & $\mathrm{M}$ & \\
\hline 24 & 19 & 25 & 43.8 & -29 & 41 & 36 & & & \\
\hline 25 & 19 & 28 & 29.0 & -30 & 8 & 55 & & M & \\
\hline 26 & 19 & 39 & 36.9 & -33 & 41 & 42 & & $\mathrm{M}$ & \\
\hline
\end{tabular}

\section{Variable Stars}

This is a report of work in progress and the observations to date are sufficient to say something about the variability of 19 of the carbon stars. Eight of them are large amplitude, $\Delta K>0.4 \mathrm{mag}$, variables, which we presume are Miras. Seven appear to be non-variable or low amplitude, $\Delta K<0.3 \mathrm{mag}$, variables although more data are required for confirmation. The other 7 require more observations to establish the character of their variations, although, as they are fainter and bluer than the others, it is unlikely that any of them is a Mira.

Periods were derived for stars number 2 and 5 , and tentative periods for stars 15 and 17; they are given in the table. The peak-to-peak variations of these Miras are about one magnitude at $J$ and smaller at longer wavelengths. A period was also derived for the SR variable with the largest number of observations, star 6. Its period is similar to those of the Miras, but its amplitude at $J$ is only half a magnitude.

It is interesting that while many carbon-rich Miras are known in the Galaxy and the MCs, these are the first carbon Miras to be identified in a dwarf galaxy. The statistics available to date suggest that 8 out of $26 \mathrm{C}$ stars, about 30 per cent of the C stars in the SDG, are Mira variables. 


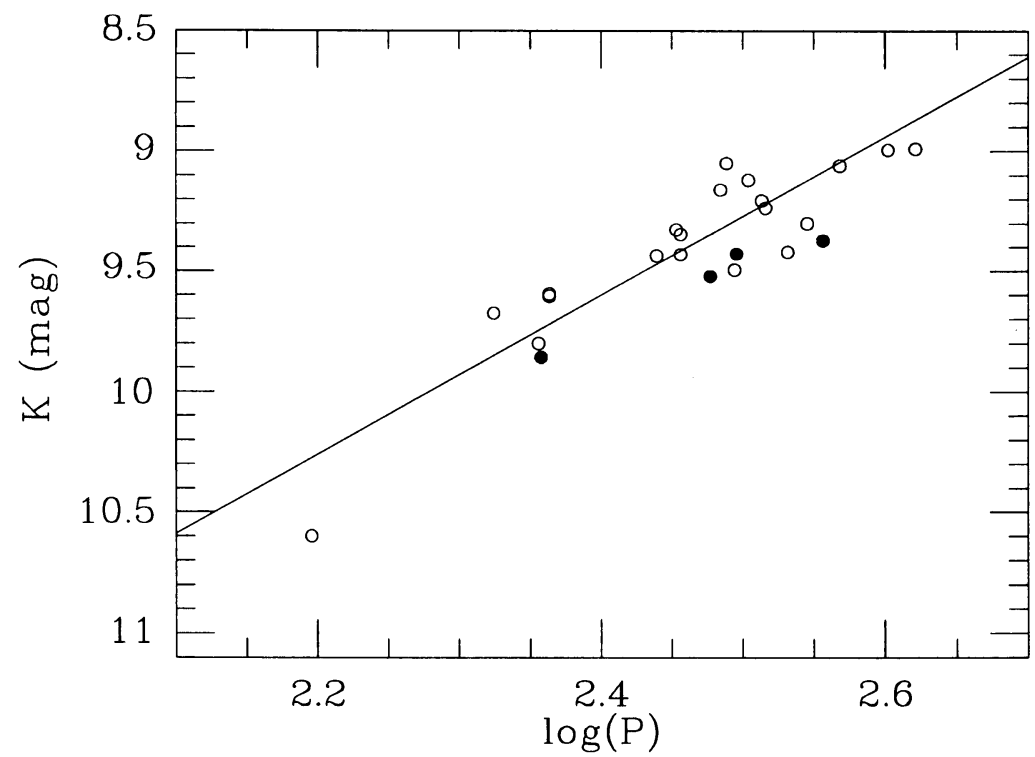

Figure 1. The PL relation; open circles are LMC Miras and closed circles are SDG Miras. The observed $K$ magnitudes are shown for the SDG Miras while the other magnitudes have been adjusted assuming the LMC and SDG distance moduli are 18.64 and 17.18 mag respectively (see text).

\section{Period Luminosity Relation and Distance of Sagittarius}

With an estimate of the period of the Miras we are in a position to compare the SDG stars with the period luminosity (PL) relation established for Miras in the LMC (Feast et al. 1989; Groenewegen \& Whitelock 1996). For this purpose we assume that the SDG has a distance modulus of $17.18 \pm 0.2$ from the Mateo et al. (1995) RR Lyr observations modified by Reid's (1998) recent work.

Figure 1 shows the LMC $\mathrm{C}$ stars and the SDG Miras on the same PL diagram. We assume that the LMC has a distance modulus of $18.64 \pm 0.13$ (derived by Whitelock et al. (1998 in preparation) from Hipparcos parallaxes for oxygen-rich Miras). We have used the LMC C star data from Feast et al. rather than those discussed by Groenewegen \& Whitelock because the Feast et al. sample contains $\mathrm{C}$ stars with multiple observations and defines a tighter $\mathrm{PL}$ relation.

The SDG Miras lie only slightly below the LMC PL relation and if we actually use them to derive the distance modulus to the SDG we get $17.36 \pm$ 0.2 , which is in good agreement with the value of $17.18 \pm 0.2$ derived from the RRs. We should emphasise that the Mira periods are tentative at this stage. Nevertheless, it looks as if there will be a sufficiently large number of Miras in the SDG that it will ultimately present us with a useful test ground for the Mira and RR Lyrae distance scales in a system somewhat less complex than the MCs. Although it would seem unlikely that these luminous AGB stars and the RRs 


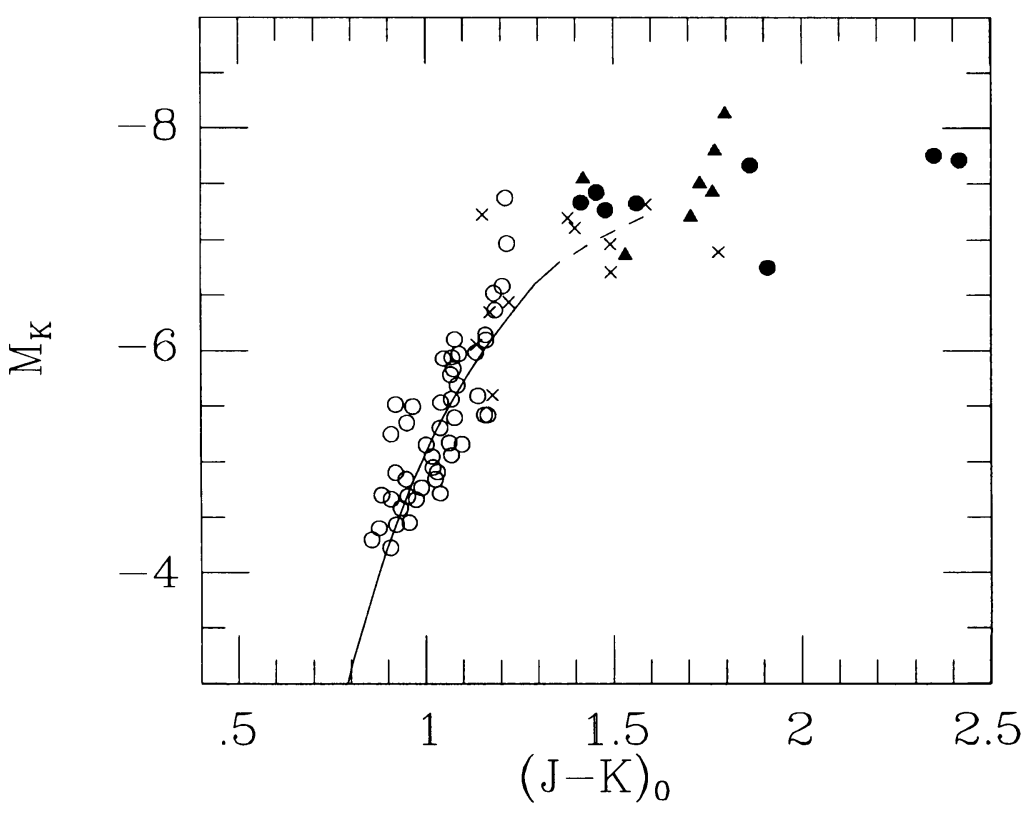

Figure 2. An infrared colour-magnitude diagram for the SDG. The open symbols represent O-rich stars from Paper I, while other symbols show C stars from this paper; circles represent Miras, triangles SRs and stars with unknown variability characteristics are shown as crosses. The 47 Tuc giant branch locus is also marked.

are from the same population, there is no reason to suspect that they will be spatially separate.

\section{Infrared Colours and Magnitudes}

An infrared colour-magnitude diagram for the SDG is shown in Fig. 2. In addition to $\mathrm{C}$ stars, distinguished according to their variability, the $\mathrm{M}$ and $\mathrm{K}$ giant branch stars from Paper I are shown. The RR distance modulus, $17.18 \mathrm{mag}$, discussed above was used in deriving the absolute magnitudes. The 47 Tuc giant branch locus from Montegriffo et al. (1995), transformed as described in Paper I, is shown for comparison; the Reid (1998) distance modulus of 13.59 mag is assumed.

A comparison of Fig. 2 with the equivalent diagram from Paper I (fig. 2) shows that the original rather blue $\mathrm{C}$ star candidates are now absent. They were either not $\mathrm{C}$ stars or were not members of SDG. The majority of the SDG $\mathrm{C}$ stars are quite clearly on the extended AGB, and provide incontrovertible evidence for an intermediate age population. The confirmed $\mathrm{C}$ stars are in fact similar to those in Fornax, although they extend to redder colours. The recently published survey of Fornax by Stetson et al. (1998) turned up some very red 


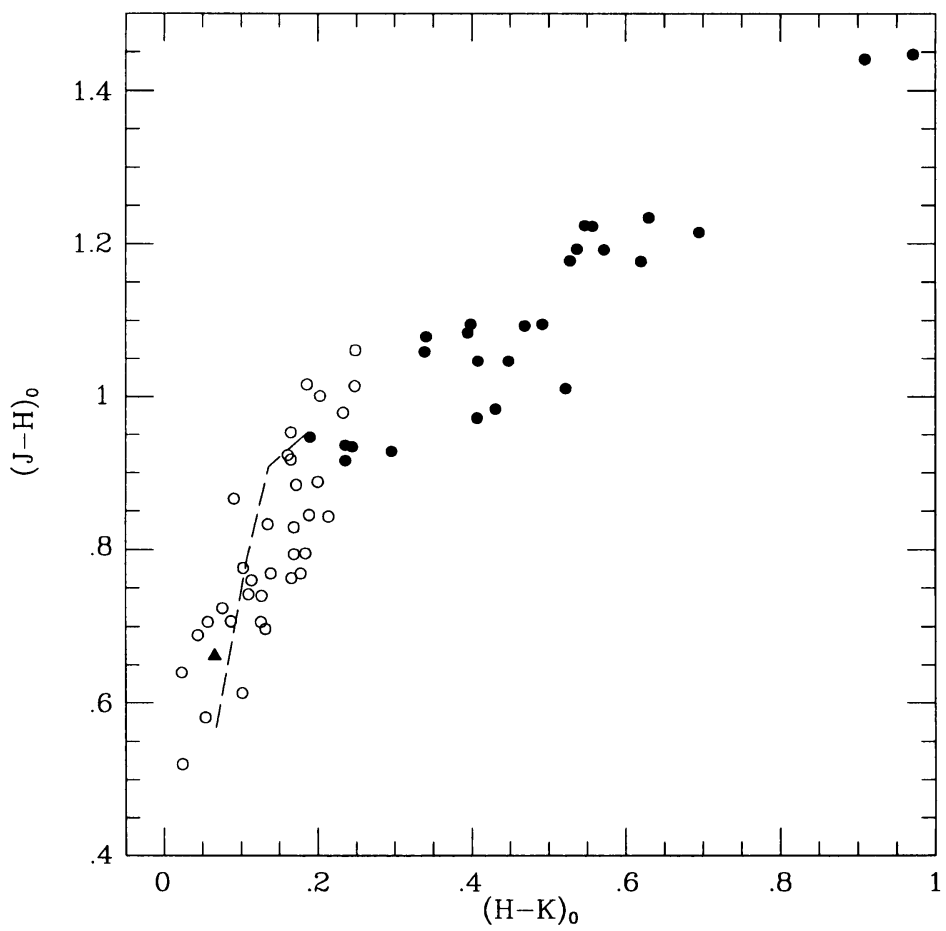

Figure 3. Two-colour diagram comparing the Bulge C stars (open circles and closed triangle) with the SDG C stars (closed circles).

C stars which may be comparable to these, but no infrared photometry has been published for them.

To a first approximation Fig. 2 translates to an HR diagram reasonably well. The bolometric correction does not change much over this colour range and temperature is roughly proportional to $J-K$. If we examine a similar diagram for a metal-rich globular cluster, the AGB is of course O-rich and does not extend so far, but the highest luminosity stars are the Miras and immediately below them are the SRs and below those the non-variable giant branch stars (see, e.g. Feast \& Whitelock 1987 fig. 1). In contrast, in the SDG the large and small amplitude variables are more or less mixed and presumably some other parameter, perhaps a range in age/mass, is the determining factor in the position of the Mira instability strip. Alternatively the distinction between C-rich Miras and SRs is not as clear as it is for O-rich stars.

\section{Carbon Stars in the Galactic Bulge}

The Bulge contains very few luminous AGB stars, a fact which can be understood by the old age and high metallicity of the Bulge population. However, Azzopardi et al. (1988) did find a few dozen low luminosity, warm, metal-rich carbon stars in the Bulge. Various, not entirely satisfactory, hypotheses have been forwarded 


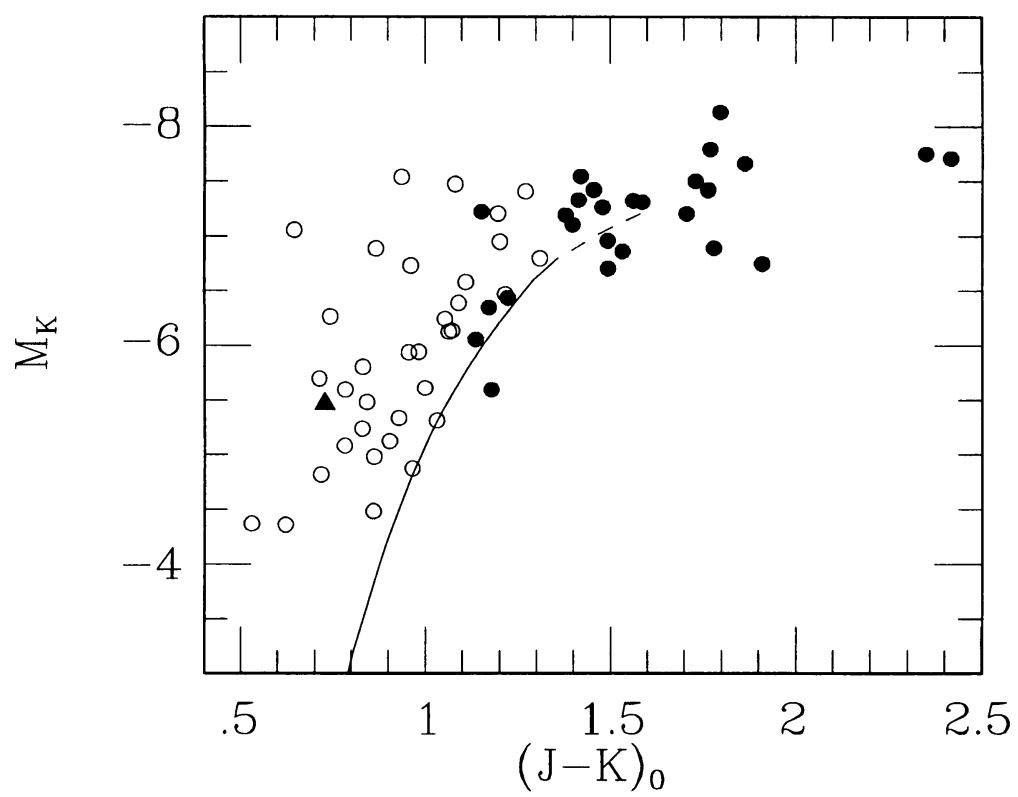

Figure 4. An infrared CMD comparing the Bulge and SDG C stars (symbols as in Fig. 3).

to explain these stars (e.g. Whitelock 1993). After the discovery of the SDG, $\mathrm{Ng}$ suggested that the $\mathrm{C}$ stars might be members of this galaxy rather than of the Bulge and he has since written several papers on the subject. In the most recent of the series $\mathrm{Ng}$ (1998) acknowledges that the Bulge C stars are indeed probably in the Bulge, but suggests they were formed during the SDG's last passage though the Bulge. For this he requires the SDG to be moving in the opposite direction to that suggested by proper motions (Irwin et al. 1996; Ibata et al. 1997; Irwin 1999), i.e. away from, rather than towards, the Bulge.

Figure 3 compares the colours of the $\mathrm{C}$ stars in the Bulge with those in SDG. For the purpose of this illustration the Azzopardi et al. (1991) data for the Bulge $\mathrm{C}$ stars was assumed to be on the ESO photometric system and converted to the SAAO system following Carter (1990). Ng suggests that the filters used by Azzopardi were not those of the ESO system discussed by Carter. In this case the conversion will not be perfect, but no plausible change in the colour equations will give the Bulge and SDG C stars the same colours. There is in fact very little overlap between the colours of the stars in these two populations. The closed triangle shows the colours of one of our SDG C star candidates that was eventually rejected because its radial velocity was inconsistent with membership. It is probably from the same Bulge population as the Azzopardi et al. Bulge stars.

Figure 4 compares the two groups in a colour-magnitude diagram. Distances were derived for the Bulge stars using Ng's (1998) formulation which assumes they were formed by the passage of SDG through the Bulge. It is clear from these diagrams that there is no relationship between the Bulge $\mathrm{C}$ stars and those 
in the SDG. This doesn't prove that the Bulge stars were not formed during the passage of the dwarf through the Bulge, but there is other evidence against that.

Acknowledgements. We are grateful to Bill Kunkel for making his observations from Las Campanas Observatory available prior to publication.

\section{References}

Azzopardi, M., Lequeux, J., Rebeirot, E. 1988, A\&A, 202, L27

Azzopardi, M., Lequeux, J., Rebeirot, E., Westerlund, B.E. 1991, A\&AS, 88, 265

Carter, B.S. 1990, MNRAS, 242, 1

Feast, M.W., Whitelock, P.A. 1987, in: Late Stages of Stellar Evolution, (eds.) S. Kwok \& S.R. Pottasch, Reidel, p. 33

Feast, M.W., Glass, I.S., Whitelock, P.A., Catchpole, R.M. 1989, MNRAS, 241, 375

Groenewegen, M.A.T., Whitelock, P.A. 1996, MNRAS, 281, 1347

Ibata, R.A., Wyse, R.F.G., Gilmore, G., Irwin, M., Suntzeff, N.B. 1997, AJ, 113,634

Ibata, R., Gilmore, G., Irwin, M.J. 1994, Nature, 370, 194

Irwin, M. 1999, these proceedings

Irwin, M.J., Ibata, R., Gilmore, G., Wyse, R., Suntzeff, N. 1996, in: The Formation of the Galactic Halo, (eds.) H. Morrison \& A. Sarjedini, ASP Conf. Ser., 92 , p. 841

Kunkel, W.E, Demers, S.D., Irwin, M.J. 1997, A\&AS, 122, 463

Mateo M., Kubiak M., Szymański M., Kałużny J., Krzemiński W., Udalski A. 1995, AJ, 110, 1141

Montegriffo, P., Ferraro, F.R., Fusi Pecci, F., Origlia, L. 1995, MNRAS, 276, 739

Ng, Y.K. 1998, A\&A, 338, 435

Reid, I.N. 1998, AJ, 115, 204

Stetson, P.B., Hesser, J.E., Smecker-Hane, T.A. 1998, PASP, 110, 533

Totten, E., Irwin, M.J. 1998, MNRAS, 294, 1

Whitelock, P.A. 1993, in: Galactic Bulges, IAU Symp. 153, (eds.) H. Dejonghe \& H.J. Habing, Kluwer, p. 39

Whitelock, P.A., Irwin, M., Catchpole, R.M. 1996, New Astro., 1, 57 